\title{
Gate control of a quantum dot single-electron spin in realistic confining potentials: anisotropy effects
}

\author{
Sanjay Prabhakar and James E. Raynolds \\ College of Nanoscale Science and Engineering, University at Albany, State University of New York, Albany, NY
}

(Dated: November 4, 2018)

\begin{abstract}
Among recent proposals for next-generation, non-charge-based logic is the notion that a single electron can be trapped and its spin can be manipulated through the application of gate potentials. In this paper, we present numerical simulations of such spins in single electron devices for realistic (asymmetric) confining potentials in two-dimensional electrostatically confined quantum dots. Using analytical and numerical techniques we show that breaking the in-plane rotational symmetry of the confining potential leads to a significant effect on the tunability of the g-factor with applied gate potentials. In particular, anisotropy extends the range of tunability to larger quantum dots.
\end{abstract}

\section{INTRODUCTION}

The notion of using single-electron spins for quantum computing and next-generation logic is an attractive idea that has received considerable attention in recent years, $1,2,3,4,5,6,7,8,9,10,11,12,13$

In order to integrate new concepts with existing semiconductor technology, a number of researchers have recently explored the possibility of using electric fields generated by imposed gate potentials to manipulate single-electron spins in quantum dot devices $14,15,16,17,18,19,20,21,22,23,24,25,26,27,28,29,30,31,32,33,34$

The goal of the present work is to utilize state-ofthe-art numerical techniques to explore the fundamental physics of single-electron spin devices and to provide realistic information for the practical design of such systems. We utilize a finite-element based numerical technique to study electrostatically defined quantum dots that is similar to other recently published work. 35

A key result of the present work is the discovery that spatial symmetry breaking $36,37,38$ resulting from the anisotropy of realistic confining potentials results in a significant enhancement of the electric-field tunability of the electron $g$-factor over that found for symmetric potentials.

\section{COMPUTATIONAL METHOD}

We utilize a multi-scale, multi-physics simulation strategy based on the finite element method ${ }^{39}$ to provide a realistic description of the physics of single-spin devices in three-dimensional geometries. The ideal is to solve selfconsistently the Maxwell equations of electrostatics with the Schroedinger equation in three dimensional geometry. Unfortunately such a solution is not feasible given currently available techniques due to the disparity of length scales in the problem. We thus seek an approximate solution that is built up in stages.

In the first step of our approach, we construct a threedimensional model of the device and calculate the gateinduced electrostatic potentials that cause the formation of a quantum dot in the two-dimensional electron gas

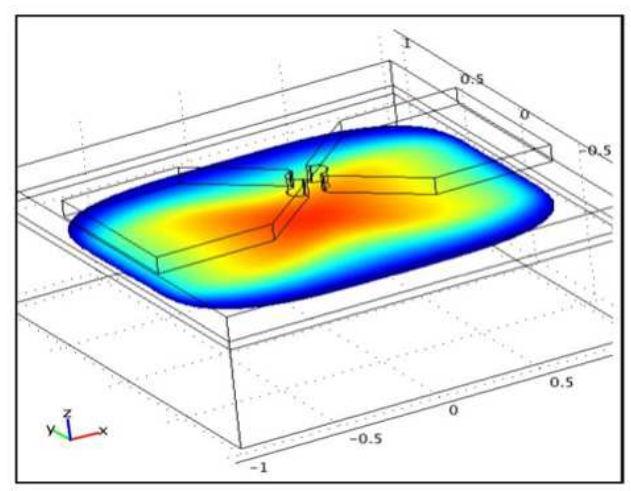

$\max 0.7 \mathrm{~V}$ 0.65

0.60

0.55

0.50

0.45

0.40

0.35

Min 0.3V

FIG. 1: (color online) Electrostatic potential for a prototype single-electron device plotted in the 2DEG layer. This figure illustrates a single-spin device consisting of two triangular gates above a 2DEG. The gates were held at $1 V$ and the $2 \mathrm{DEG}$ was held at $0 \mathrm{~V}$. For simplicity of the electrostatic calculation, the 2DEG was treated as a classical perfect conductor. The dimensions of the device in the $x$ and $y$ directions are $2.8 \mu \mathrm{m}$ and $1.8 \mu \mathrm{m}$ respectively and the thickness is $1 \mu \mathrm{m}$.

(2DEG) at a AlGaAs/GaAs heterojunction as illustrated in Figs. 1, 2] and 3. This geometry corresponds to prototype devices that are under consideration by experimentalists at the University at Albany, State University of New York.

In order to obtain the electrostatic solution for the confining potential, we approximate the 2DEG as a classical perfect conductor and give it a finite width. The width $(\approx 0.05 \mu \mathrm{m})$ is unrealistically large from a quantum perspective but is assumed to give a reasonable description of the spatial variation of the potential in the layer of the 2DEG. In a subsequent step we treat the 2DEG from a realistic quantum mechanical perspective. 40

Figs. 2] and 3 are one-dimensional plots obtained from Fig. 1 by plotting the potential along a line in the 2DEG along high symmetry directions. These one-dimensional potentials are then fit to polynomial forms $P_{x}(x)$ and 


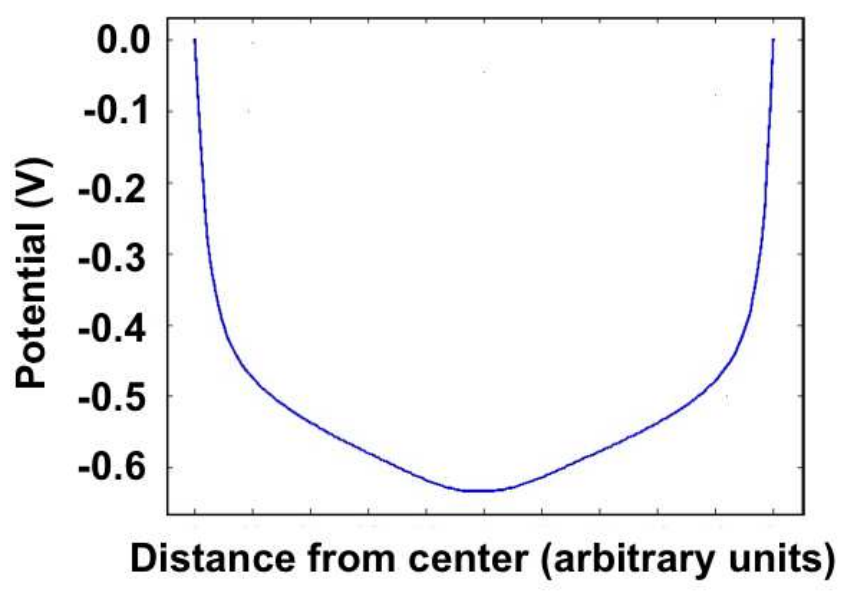

FIG. 2: Electrostatic confining potential in the 2DEG along the symmetry axis of a prototype single-electron device. This figure was made by plotting the potential of Fig. 1 along a line in the 2DEG through the symmetry axis of the device (the $\mathrm{x}$-axis of Fig. 1 i.e. a line running from one gate to the other) intersecting with the central region.

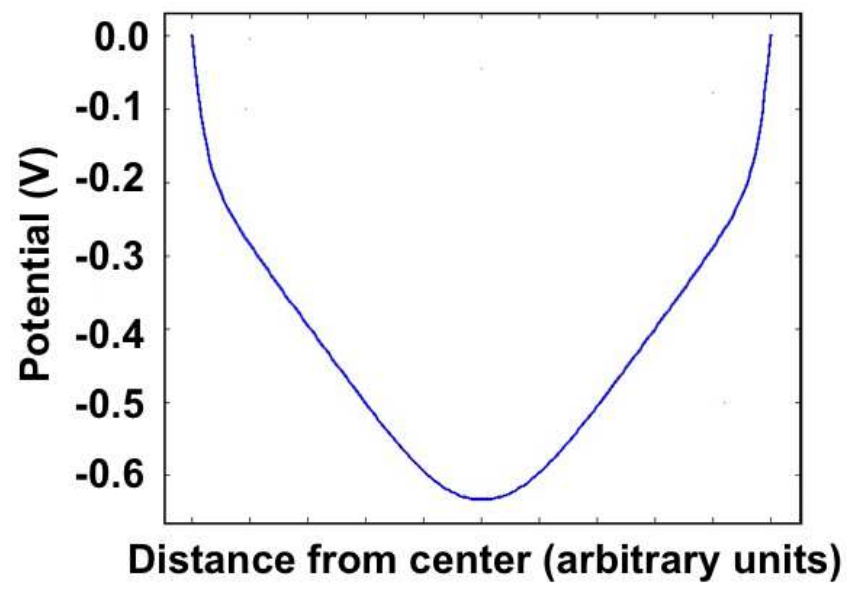

FIG. 3: Electrostatic confining potential in the 2DEG normal to the symmetry axis of a prototype single-electron device. This figure was made by plotting the potential of Fig. 1 1along a line in the 2DEG normal to the symmetry axis of the device (the y-axis of Fig. 1) and intersecting the central region.

$P_{y}(y)$. These are then used as a potential of the form

$$
V_{\text {real }}(x, y) \approx P_{x}(x)+P_{y}(y)
$$

to approximate the confining potential of the electron in the Schroedinger equation. Before considering electron motion in the above potential, $V_{\text {real }}$, we consider the simpler quadratic potential

$$
V_{\text {quad }} \equiv \frac{1}{2} m \omega_{o}^{2}\left(\alpha x^{2}+\beta y^{2}\right)
$$

that allows for systematic studies. For convenience we have written the strength of the potential in harmonic oscillator form by defining the pre-factor $\frac{1}{2} m \omega_{o}^{2}$.

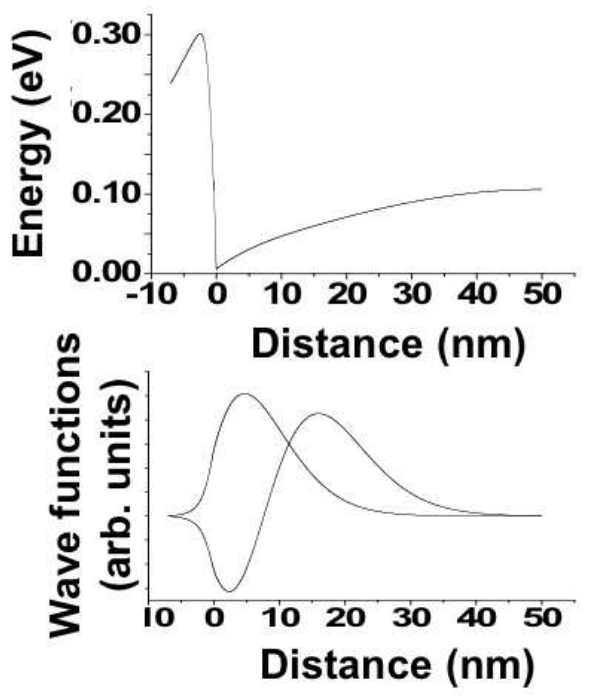

FIG. 4: Heterojunction self-consistent potential and lowest two wave functions of the 2DEG. These results demonstrate consistency of our results with other published work..$^{40}$

In the second step we calculate the wave functions and self-consistent potential at the heterojunction between AlGaAs and GaAs that describes the formation of the two dimensional electron gas as illustrated in Fig. 4. We do this calculation primarily to benchmark our numerical method by making contact with a well-know result from the literature $\underline{\underline{40}}$

The results of Fig. 4 are obtained by solving the following coupled equations that constitute the self-consistent Schroedinger-Poisson equations including exchange-correlation effects

$$
\begin{gathered}
\frac{-\hbar^{2}}{2} \frac{d}{d z}\left(\frac{1}{m(z)} \frac{d \psi_{i}(z)}{d z}\right)+V(z) \psi_{i}(z)=E_{i} \psi_{i}(z) \\
\frac{d}{d z}\left(\epsilon_{o} \kappa(z) \frac{d \phi(z)}{d z}\right)=e \sum_{i} n_{i}\left|\psi_{i}(z)\right|^{2}-\rho(z)
\end{gathered}
$$

where $\kappa(z)$ and $\rho(z)$ are the fixed spatially dependent dielectric function and background charge density of the the interface as described in Ref. 40. The potential energy of the $2 \mathrm{DEG}$ is given by:

$$
V(z)=-e \phi(z)+V_{x c}(z)
$$

where $\phi(z)$ is the self-consistent potential and $V_{x c}(z)$ is the exchange-correlation potential. In the above equations, the coordinate $z$ is measured relative to the interface between AlGaAs and GaAs.

The results of Fig. 4 are in excellent agreement with previous results 40 confirming the soundness of our approach.

We next consider the formation of an electrostatically defined quantum dot by applying a symmetric, confining 


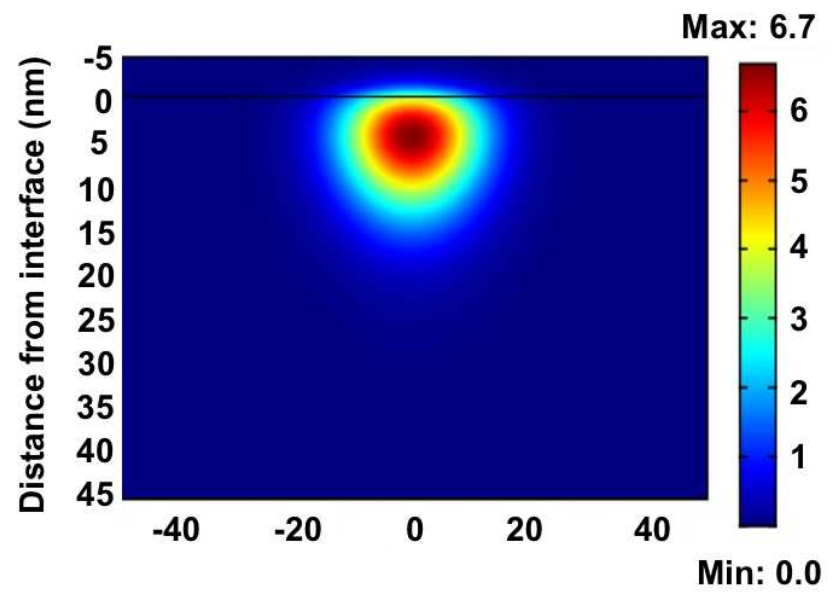

FIG. 5: (color online) Quantum dot wave function plotted in the $\mathrm{X}-\mathrm{z}$ plane formed by applying a quadratic confining potential in the plane (i.e. along the $x$ axis). This potential is characterized by the parameter $\ell_{0}=20 \mathrm{~nm}$ (quantum dot radius, see Eq. 16).

potential in the plane of the 2DEG as illustrated in Fig.5. In other words, we add a potential of the form

$$
V_{x}(x)=\frac{1}{2} m \omega_{o}^{2} x^{2}
$$

to Eq. 5 and solve the system of Eqs. 3, 4, and 5] selfconsistently in the two-dimensional $x-z$ domain. Figure 5 clearly illustrates the formation of a quantum dot in the potential well of the 2DEG as expected.

In the remainder of this paper we focus our attention on motion in the plane of the 2DEG and contrast effects associated with quantum dots in symmetric and asymmetric confining potentials as illustrated in Figs. 6 and 7, respectively. These figures were obtained using the quadratic model potential of Eq. 2 with $\alpha=\beta=1$ and $\alpha=1 ; \beta=2.8$ respectively in the two-dimensional inplane (i.e. $x-y$ plane) Hamiltonian, $H_{x y}$, to be discussed in the following. The parameters of the asymmetric potential were chosen so as to mimic the realistic potential of Figs. 2] and 3. The wave function in the asymmetric model potential of Fig. 7 should be contrasted with the wave function in the realistic potential (i.e. using the form of Eq. 1) as shown in Fig. 8 .

We consider the motion of the electron in the $x-y$ plane of the quantum dot in the presence of a magnetic field oriented perpendicular to the plane of the 2DEG. Our approach closely follows that of Ref. 14. Thus the total Hamiltonian can be written as

$$
H=H_{x y}+H_{z}+H_{s o}
$$

where $H_{z}$ corresponds to motion normal to the interface (as discussed in the context of Eqs. 3, 4] and 5), $H_{s o}$ is the spin-orbit interaction to be discussed shortly and the remaining term is given by:

$$
H_{x y}=\frac{\vec{P}^{2}}{2 m}+\frac{1}{2} m \omega_{o}^{2}\left(\alpha x^{2}+\beta y^{2}\right)+g_{o} \mu_{B} \sigma_{z} B
$$

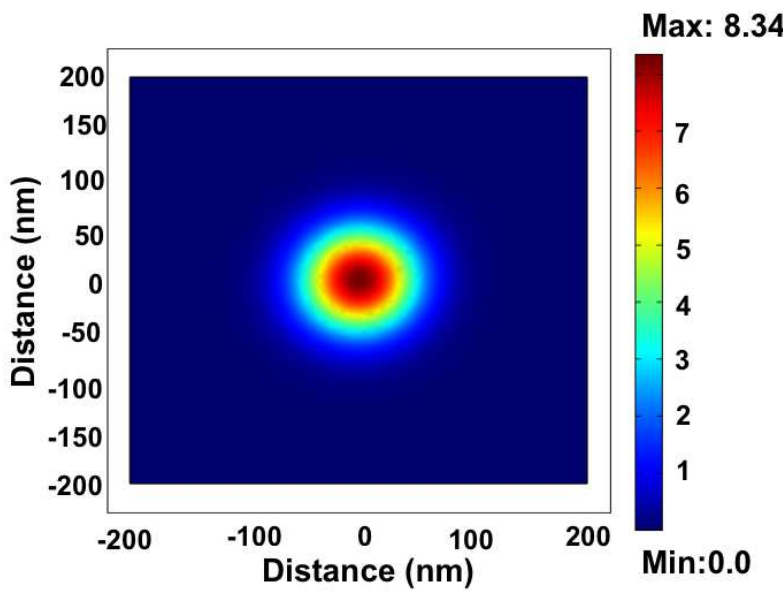

FIG. 6: (color online) In-plane wave-function for quantum dot formed by a symmetric quadratic confining potential (Eq. 2) in the $(x-y)$ plane with $\ell_{o}=40 \mathrm{~nm}$ (see Eq. 16).

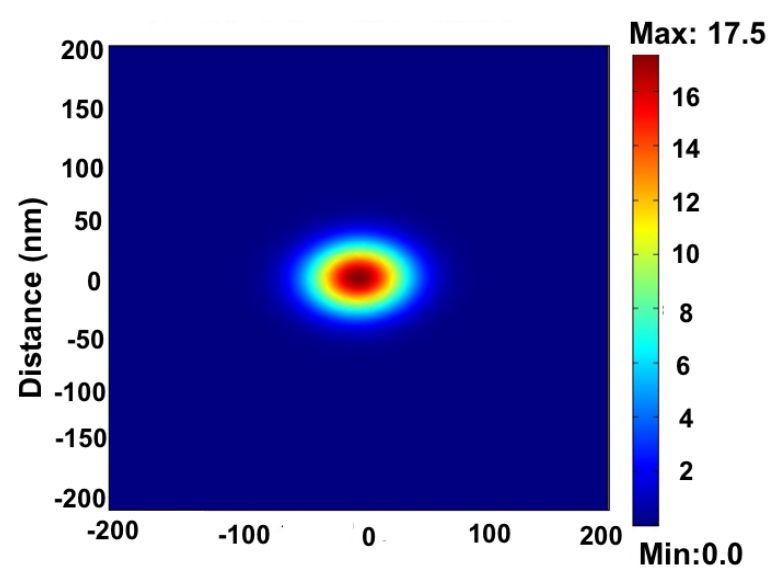

FIG. 7: (color online) In-plane wave-function for quantum dot formed by an asymmetric quadratic confining potential in the $(x-y)$ plane. The parameters for this potential where chosen to mimic the realistic potential of Figs. 2 and 3 respectively and are given by $\ell_{o}=30 \mathrm{~nm}$ (see Eq. 16) and $\alpha=1$ and $\beta=2.8$ (see Eq. 2).

where the kinetic momentum operator:

$$
\vec{P} \equiv \vec{p}+\frac{e}{c} \vec{A}
$$

is the sum of the canonical momentum

$$
\vec{p} \equiv-i \hbar\left(\partial_{x}, \partial_{y}, 0\right)
$$

and the vector potential (in the symmetric gauge)

$$
\vec{A} \equiv \frac{B}{2}(-y, x, 0)
$$

The eigenstates of $H_{x y}$ (Eq. 8) with $\alpha=\beta$ are the wellknown Fock-Darwin states $\frac{41,42}{43}$ The situation with $\alpha \neq \beta$ also has an analytic solution. 43 We have verified that our 


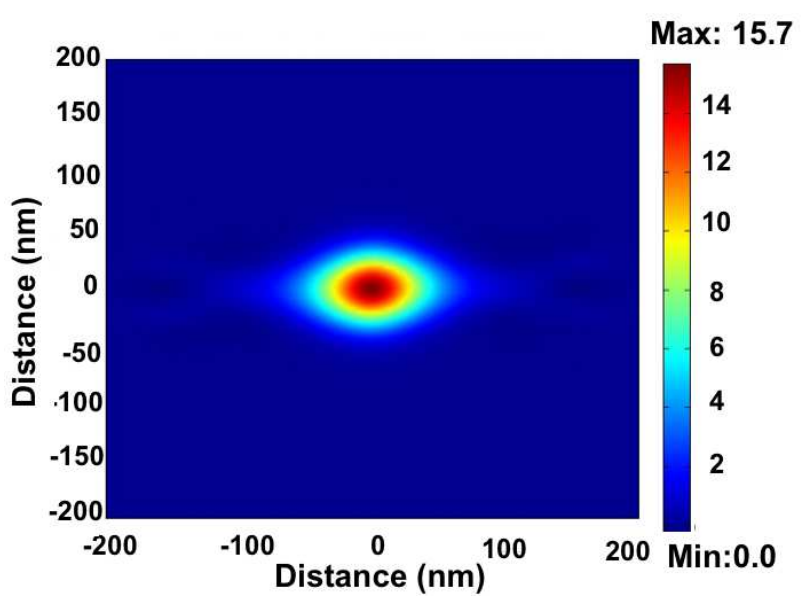

FIG. 8: (color online) In-plane wave-function for quantum dot formed by the potential of Eq. 1 1 illustrated in Figs. 2 and 3

numerical solution of $H_{x y}|\psi>=\epsilon| \psi>$ is consistent with these analytical results.

Lastly we consider the spin orbit interaction as embodied in the Hamiltonian $H_{s o}$ which is the essential ingredient in the phenomena of electric field induced spin switching $\underline{6,14}$ We write:

$$
H_{s o}=H_{R}+H_{D 1}+H_{D 2}
$$

where the Rashba interaction 44,45 is given by:

$$
H_{R}=\frac{\alpha_{R} e E}{\hbar}\left(\sigma_{x} P_{y}-\sigma_{y} P_{x}\right)
$$

and the linear and cubic Dresselhous interactions ${ }^{46.47}$ are written as:

$$
H_{D 1}=\frac{0.7794 \gamma_{c} k^{2}}{\hbar}\left(-\sigma_{x} P_{x}+\sigma_{y} P_{y}\right),
$$

which is linear in components of the momentum operator $\vec{P}$ and

$$
H_{D 2}=\frac{\gamma_{c}}{\hbar^{3}}\left(-\sigma_{x} P_{x} P_{y}^{2}-\sigma_{y} P_{y} P_{x}^{2}\right)+h . c .,
$$

which is cubic in components of the momentum operator (h.c. denotes the Hermitian conjugate) 14 Note, the electric field strength $E$ that enters Eq. 13 is that associated with the heterojunction $|E|=\partial V(z) / \partial z$ and is treated as an adjustable parameter. Physically we can implement changes in $E$ through the application of appropriate gate potentials. All numerical parameters in the above pieces of $H_{s o}$ are those for GaAs found in Ref. 14

The eigenvalue equation $H|\psi>=\epsilon| \psi>$, with $H$ given by Eqs. 7 through [15, was solved numerically to obtain the lowest few eigenstates and eigenenergies vs. the various parameters of the system. These parameters include the magnetic field strength $B$, the electric field $E$, and the strength of the quantum dot confinement potential. This latter parameter is conveniently characterized by defining the quantum dot radius as follows:

$$
\ell_{0} \equiv \sqrt{\frac{\hbar}{m \omega_{o}}}
$$

The notion of electric field induced spin switching is quantified by defining an effective electron $g$ factor by the following definition:

$$
\epsilon=\frac{1}{2} g \mu_{B} \sigma_{z} B
$$

to describe the energy difference between the lowest energy up and down spin states. Thus we consider the lowest two states (including spin) $\epsilon_{2}$ and $\epsilon_{1}$ and calculate the effective $g$ factor as:

$$
g=\frac{\left(\epsilon_{2}-\epsilon_{1}\right)}{\mu_{B} B} .
$$

Results for the variation of this effective $g$ factor as a function of the parameters $E, B$ and $\ell_{o}$ is presented in the following section.

\section{RESULTS}

We now turn to a presentation of the key results of this work: the tunability of the electron $g$-factor through the application of electric and magnetic fields.

Figure 9 is consistent with previous published work ${ }^{14}$ and illustrates the $g$-factor tunability vs. the strength of the applied electric field and confining potential (as parametrized by the quantum dot radius $\ell_{o}$ ) for fixed magnetic field $(B=1 T)$ for the symmetric quantum dot in the quadratic potential of Eq. 2 with $\alpha=\beta=1$.

Figure 10 is also consistent with previous published work $^{14}$ and illustrates the $g$-factor tunability vs. the strength of the applied electric field and and magnetic field for fixed confining potential (parametrized by the quantum dot radius $\left.\ell_{o}=20 \mathrm{~nm}\right)$.

Upon introducing in-plane anisotropy to the confining potential we find significant changes in the electric-field induced $g$-factor tunability as illustrated in Fig. 11. This figure was generated by choosing $\alpha=1, \beta=2$ and $\ell_{o}=$ $120 \mathrm{~nm}$.

To quantify the effects of in-plane anisotropy, we have carried out a parameter study of the $g$-factor tunability vs. degree of anisotropy and the results are presented in Fig. 12. This figure was generated by fixing the quantum dot radius at $\ell_{o}=120 \mathrm{~nm}$ and holding $\alpha=1$ while varying $\beta$ with $B=1 T$.

Lastly we consider the results for $g$-factor tunability for quantum dots in the realistic potential of Figs. 2 and 3 and Eq.1 Figure13illustrates the results for a quantum dot in the realistic potential (middle curve) in comparison with symmetric (lower curve) and asymmetric (upper curve) quantum dots. The parameters were chosen 


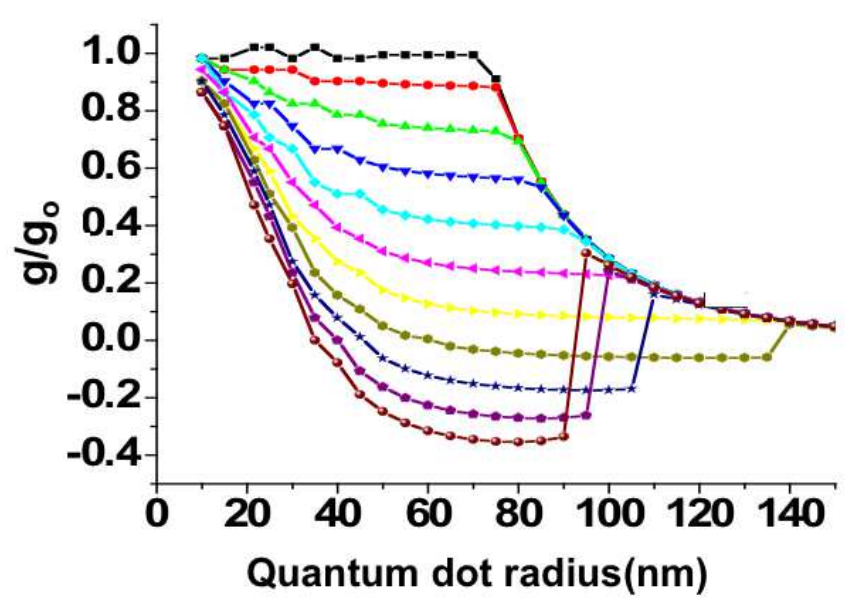

FIG. 9: (color online) Electric field induced changes in the $g$-factor vs. quantum dot radius for various electric field strengths for a symmetric quantum dot in the quadratic potential of Eq. 2 From top to bottom, the curves represent increasing electric field strength as follows. The first curve corresponds to $1 \times 10^{4} \mathrm{~V} / \mathrm{cm}$, and the rest range from $1 \times 10^{5} \mathrm{~V} / \mathrm{cm}$ through $1 \times 10^{6} \mathrm{~V} / \mathrm{cm}$ in equal steps with $B=1 T$. This results is consistent with Ref. 14 .

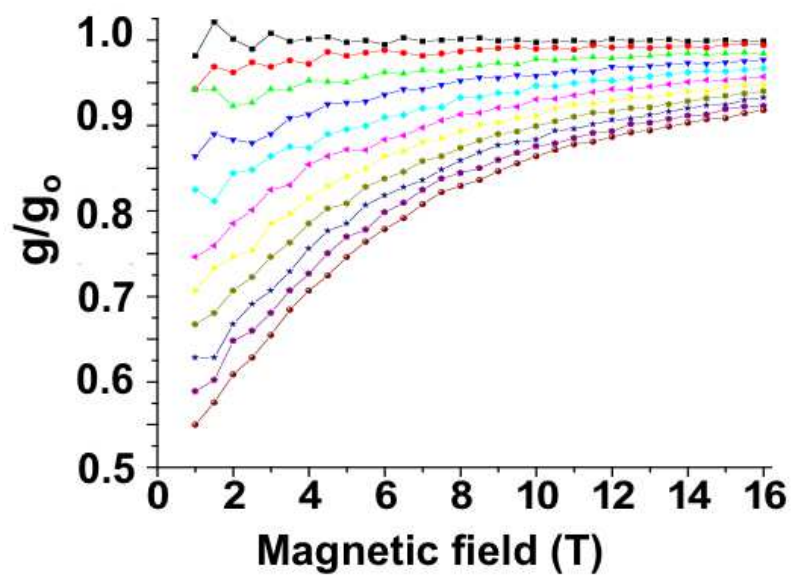

FIG. 10: (color online) Electric field induced changes in the $g$ factor vs. magnetic field for various electric field strengths for the symmetric quantum dot. From top to bottom, the curves represent increasing electric field strength as follows. The first curve corresponds to $1 \times 10^{4} \mathrm{~V} / \mathrm{cm}$, and the rest range from $1 \times 10^{5} \mathrm{~V} / \mathrm{cm}$ through $1 \times 10^{6} \mathrm{~V} / \mathrm{cm}$ in equal steps. For this calculation, the quantum dot radius was fixed at $\ell_{o}=20 \mathrm{~nm}$

to closely mimic the realistic potential and are given by $\ell_{o}=30 \mathrm{~nm}$ and $\alpha=\beta=1$ for the symmetric potential and $\alpha=1 ; \beta=2.8$ for the asymmetric potential.

From Fig. 13 we conclude that the realistic potential result is bracketed by those for symmetric and asymmetric model potentials and, as such, contains characteristics of each.

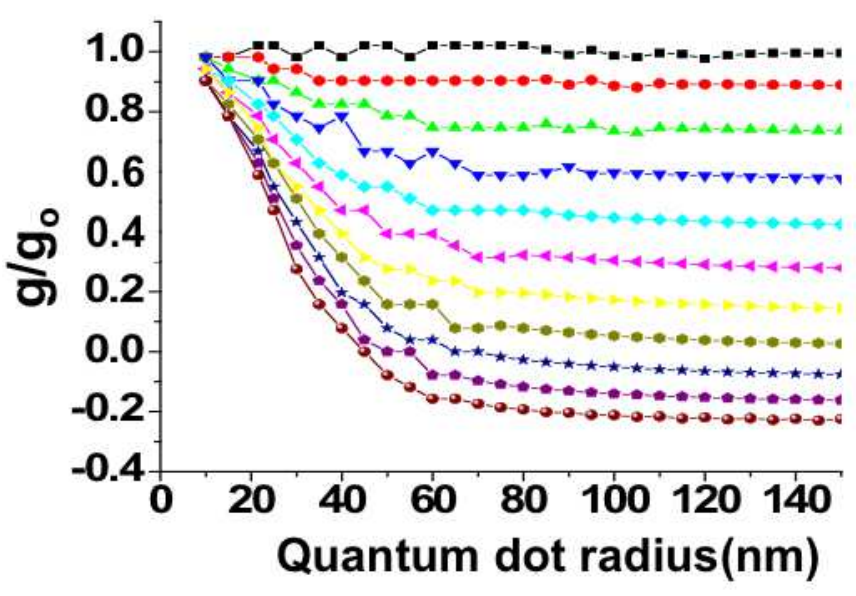

FIG. 11: (color online) Electric field induced changes in the $g$-factor vs. quantum dot radius for various electric field strengths for an asymmetric quantum dot (wave function not shown). From top to bottom, the curves represent increasing electric field strength as follows. The first curve corresponds to $1 \times 10^{4} \mathrm{~V} / \mathrm{cm}$, and the rest range from $1 \times 10^{5} \mathrm{~V} / \mathrm{cm}$ through $1 \times 10^{6} \mathrm{~V} / \mathrm{cm}$ in equal steps. Again we choose $B=1 T$.

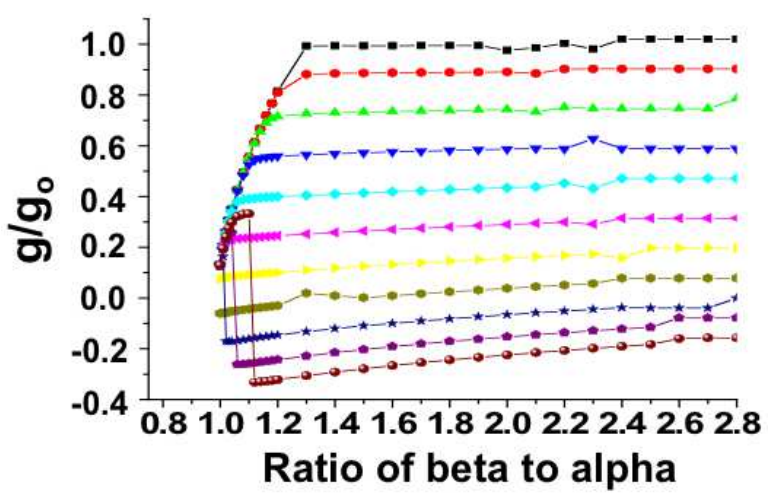

FIG. 12: (color online) Electic field induced changes in the $g$ factor vs. the degree of anisotropy of the quantum dot confinement potential for various electric field strengths. From top to bottom, the curves represent increasing electric field strength as follows. The first curve corresponds to $1 \times 10^{4} \mathrm{~V} / \mathrm{cm}$, and the rest range from $1 \times 10^{5} \mathrm{~V} / \mathrm{cm}$ through $1 \times 10^{6} \mathrm{~V} / \mathrm{cm}$ in equal steps.

\section{DISCUSSION}

The key result of this work is illustrated in Figs. 9 and 11] anisotropy in the confining potential significantly extends the size range of quantum dots that exhibit electric field induced $g$-factor tunability. Indeed, in Fig. 9 we see that all of the curves collapse onto a single curve for large quantum dots (i.e. larger than $\ell_{o}=120 \mathrm{~nm}$ ) negating the switching effect. With anisotropy, however, there is no degradation of the switching effect for large dots.

Another important result of this work is the realization that the degree of anisotropy need not be very large in 


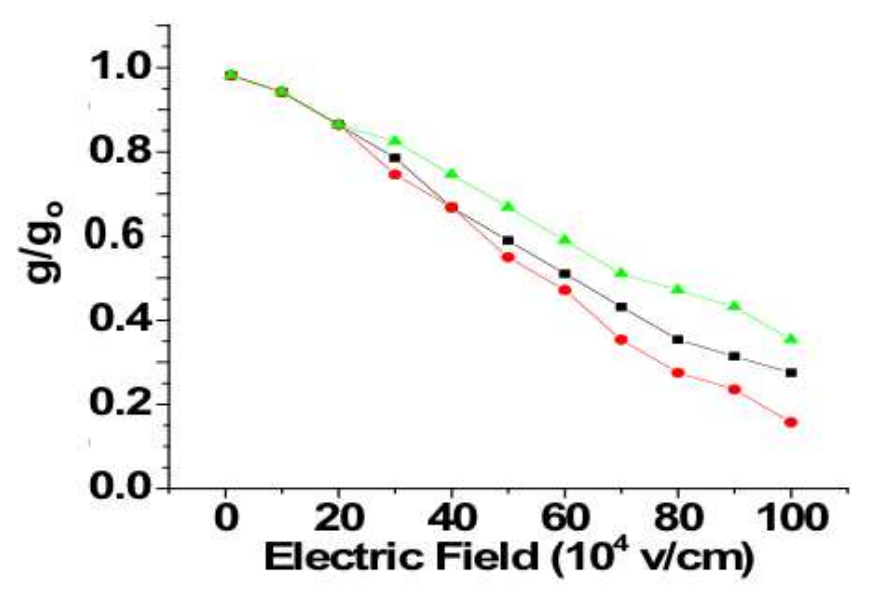

FIG. 13: (color online) Changes in the $g$-factor vs. electric field for quantum dots in the realistic potential of Figs. 2 and 3 (using the form of Eq. 1) in comparison to results for symmetric and asymmetric model potentials. The black (middle) curve corresponds to the realistic potential while the red (lower) and green (upper) curves correspond to symmetric and asymmetric model potentials respectively. The parameters were chosen to closely mimic the realistic potential and are given by $\ell_{o}=30 \mathrm{~nm}$ and $\alpha=\beta=1$ for the symmetric potential and $\alpha=1 ; \beta=2.8$ for the asymmetric potential.

order to obtain significant changes in the gate induced $g$-factor tunability, as illustrated in Fig. 12, We see that the maximum effect is obtained for roughly $\beta / \alpha \approx 1.3$ and begins to decrease slightly for larger values of this shape-anisotropy ratio. The jumps in the value of the $g$-factor from positive to negative value are indicative of level crossings (e.g. the relative ordering of spin up and down levels changes as a function of the anisotropy).

Lastly we have seen from Fig. 13 that results for quantum dots in realistic potentials contain characteristics of both the symmetric and asymmetric model potentials. This emphasizes the need for realistic numerical simulations since the physics of such systems cannot be fully captured by symmetric or asymmetric model potentials alone.

\section{CONCLUSIONS}

We have carried out a numerical simulation study of gate induced tunability of the electron $g$-factor in a prototype single electron spintronic device. We consider a realistic three-dimensional geometry and employ a numerical approach based on the finite element method ${ }^{39}$.

Due to the large disparity in physical length scales we have adopted an approximate strategy as a complete selfconsistent solution of the full problem is prohibitive given existing computational tools. In our approach we have investigated the problem in stages.

We find that symmetry breaking due to anisotropy of the quantum dot confining potential leads to significant changes in the gate induced tunability of the $g$-factor vs. quantum dot radius extending the size range of quantum dots that can be tuned. We also find that the anisotropy ratio need only be on the order of $\beta / \alpha=1.3$ to produce the largest effects.

By employing non-perturbative, fully numerical methods and realistic geometries, our approach is providing insights that might be difficult or impossible to obtain using analytical techniques alone.
1 D. Loss and D. P. DiVincenzo, Phys. Rev. A 57, 120 (1998).

${ }^{2}$ H.-A. Engel, L. P. Kouwenhoven, D. Loss, and C. M. Marcus, Quantum Information Processing 3, 115 (2004).

3 W. A. Coish and D. Loss, Phys. Rev. B 75, 161302 (2007).

${ }^{4}$ D. D. Awschalom, D. Loss, and N. Samarth, eds., Semiconductor Spintronics and Quantum Computation (Springer, Berlin, 2002).

5 R. Hanson, L. P. Kouwenhoven, J. R. Petta, and S. Tarucha, Rev. Mod. Phys. 79, 1217 (2007).

6 I. Zutic, J. Fabian, and S. D. Sarma, Rev. Mod. Phys. 76, 323 (2004).

7 S. Bandyopadhyay, Phys. Rev. B 61, 13813 (2000).

8 A. R. Trivedi and S. Bandyopadhyay, J. Appl. Phys. 103, 104311 (2008).

9 T. Calarco, A. Datta, P. Fedichev, E. Pazy, and P. Zoller, Phys. Rev. A 68, 012310 (2003).

10 R. Hanson and D. D. Awschalom, Nature 453, 1043 (2008).

11 E. Biolatti, I. D'Amico, P. Zanardi, and F. Rossi, Phys. Rev. B 65, 075306 (2002).

12 D. K. Young, J. A. Gupta, E. Johnston-Halperin, R. Ep- stein, Y. Kato, and D. D. Awschalom, Semicond. Sci. Tech. 17, 275 (2002).

13 M. Thorwart and P. Hangg, Phys. Rev. A 65, 012309 (2001).

14 R. de Sousa and S. D. Sarma, Phys. Rev. B 68, 155330 (2003).

15 E. I. Rashba and A. L. Efros, Appl. Phys. Lett. 83, 5259 (2003).

16 E. A. Laird, C. Barthel, E. I. Rashba, C. M. Marcus, M. P. Hanson, and A. C. Gossard, Phys. Rev. Lett. 99, 246601 (2007).

17 C. S. Tang, A. G. Malshukov, and K. A. Chao, Phys. Rev. B 71, 195314 (2005).

18 J. Pingenot, C. E. Pryor, and M. E. Flatte, Appl. Phys. Lett. 92, 222502 (2008).

19 H. W. Jiang and E. Yablonovitch, Phys. Rev. B 64, 041307 (2001).

20 Y.-P. Shim and P. Hawrylak, Phys. Rev. B 78, 165317 (2008).

21 K. C. Nowack, F. H. L. Koppens, Y. V. Nazarov, and L. M. K. Vandersypen, Science 318, 1430 (2007).

22 A. L. Efros, E. I. Rashba, and M. Rosen, Phys. Rev. Lett. 
87, 206601 (2001).

23 S. Tarucha and J. Baugh, J. Phys. Soc. Japan 77, 031011 (2008).

24 S. Amasha, K. MacLean, I. P. Radu, D. M. Zumbuhl, M. A. Kastner, M. P. Hanson, and A. C. Gossard, Phys. Rev. Lett. 100, 046803 (2008).

25 J.-M. Tang, J. Levy, and M. E. Flatte, Phys. Rev. Lett. 97, 106803 (2006).

${ }^{26}$ G. Ortner, M. Bayer, Y. Lyanda-Geller, T. L. Reinecke, A. Kress, J. P. Reithmaier, and A. Forchel, Phys. Rev. Lett. 94, 157401 (2005).

27 G. Bester and A. Zunger, Phys. Rev. B 72, 165334 (2005).

28 A. Hoegele, S. Seidl, M. Kroner, K. Karrai, R. J. Warburton, M. Atatre, J. Dreiser, A. Imamoglu, B. D. Gerardot, and P. M. Petroff, J. Supercond. 18, 245 (2005).

29 L. S. Levitov and E. I. Rashba, Phys. Rev. B 67, 115324 (2003).

30 M. Governale, F. Taddei, and R. Fazio, Phys. Rev. B 68, 155324 (2003).

31 T. Nakaoka, S. Tarucha, and Y. Arakawa, Phys. Rev. B 76, 041301 (2007).

${ }^{32}$ M. F. Doty, M. Scheibner, I. V. Ponomarev, E. A. Stinaff, A. S. Bracker, V. L. Korenev, T. L. Reinecke, and D. Gammon, Phys. Rev. Lett. 97, 197202 (2006).
33 Y. K. Kato and D. D. Awschalom, J. Phys. Soc. Japan 77, 031006 (2008).

34 E. I. Rashba and A. L. Efros, Phys. Rev. Lett. 91, 126405 (2003).

35 S. Bednarek, K. Lis, and B. Szafran, Phys. Rev. B 77, 115320 (2008).

36 A. Kwasniowski and J. Adamowski, J. Phys. Cond. Matt. 20, 215208 (2008).

37 J. Konemann, R. J. Haug, D. K. Maude, V. I. Falko, and B. L. Altshuler, Phys. Rev. Lett. 94, 226404 (2005).

38 C. F. Destefani and S. E. Ulloa, Phys. Rev. B 71, 161303 (2005).

39 Comsol Multiphysics version 3.4 (www.comsol.com).

40 F. Stern and S. D. Sarma, Phys. Rev. B 30, 840 (1984).

41 V. Fock, Physik 47, 446 (1928).

42 C. G. Darwin, Proc. Cambridge Philos. Soc. 27, 86 (1930).

43 B. Schuh, J. Phys. A: Math. Gen. 18, 803 (1985).

44 E. I. Rashba, Fiz. Tverd. Tela (Lenningrad) 2, 1224 (1960).

45 Y. A. Bychkov and E. I. Rashba, J. Phys. C 17, 6039 (1984).

46 G. Dresselhaus, Phys. Rev. 100, 580 (1955).

47 M. Dyakonov and V. Kachorovskii, Fiz. Tekh. Poluprovodn. (S.-Petersberg) 20, 178 (1986). 